FACTA UNIVERSITATIS (NIŠ)

Ser. Math. Inform. Vol. 35, No 5 (2020), 1251-1258

https://doi.org/10.22190/FUMI2005251S

\title{
ARENS REGULARITY OF PROJECTIVE TENSOR PRODUCT
}

\author{
Mostfa Shams Kojanaghi and Kazem Haghnejad Azar
}

(C) 2020 by University of Niš, Serbia | Creative Commons Licence: CC BY-NC-ND

Abstract. In this paper, we study some of approximate identity properties, and its application in the Arens regularity of tensor products of Banach algebras with some results in group algebras. W e consider under which sufficient and necessary conditions the Banach algebra $A \widehat{\otimes} B$ is Arens regular.

Keywords: Arens regularity; tensor product; Banach algebras; group algebras.

\section{Introduction}

Suppose that $A$ and $B$ are Banach algebras. Since 1988 the Arens regularity of $A \widehat{\otimes} B$ has received a great deal of attention by many researchers. Among them, Ülger in $[19,21]$ showed that $A \widehat{\otimes} B$ is not Arens regular, in general, even when $A$ and $B$ are Arens regular. He introduced a new concept of biregular mapping and showed that a bounded bilinear mapping $m: A \times B \rightarrow \mathbb{C}$ is biregular if and only if $A \widehat{\otimes} B$ is Arens regular, where $\mathbb{C}$ is the space of complex numbers. Let $X, Y$ and $Z$ be normed spaces and let $m: X \times Y \rightarrow Z$ be a bounded bilinear mapping. Arens in [1] offers two natural extensions $m^{* * *}$ and $m^{t * * * t}$ of $m$ from $X^{* *} \times Y^{* *}$ into $Z^{* *}$ that he called $m$ is Arens regular whenever $m^{* * *}=m^{t * * t}$, for more information see $[9,10,14]$. Let $A$ be a Banach algebra, regarding $A$ as a Banach $A$-bimodule, the operation $\pi: A \times A \longrightarrow A$ extends to $\pi^{* * *}$ and $\pi^{t * * * t}$ defined on $A^{* *} \times A^{* *}$. These extensions are known, respectively, as the first (left) and the second (right) Arens products, and with each of them, the second dual space $A^{* *}$ becomes a Banach algebra. The regularity of a normed algebra $A$ is defined to be the regularity of its algebra multiplication when considered as a bilinear mapping. The first (left) and second (right) Arens products of $a^{\prime \prime}, b^{\prime \prime} \in A^{* *}$ shall be simply indicated by $a^{\prime \prime} b^{\prime \prime}$ and $a^{\prime \prime} o b^{\prime \prime}$, respectively. Let $B$ be a Banach $A$-bimodule, and let

$$
\pi_{\ell}: A \times B \longrightarrow B \text { and } \pi_{r}: B \times A \longrightarrow B,
$$

Received January 04, 2020; accepted March 09, 2020

2020 Mathematics Subject Classification. Primary 46B40; Secondary 32A65 
be the right and left module actions of $A$ on $B$. By above notation, the transpose of $\pi_{r}$ denoted by $\pi_{r}^{t}: A \times B \rightarrow B$. Then

$$
\pi_{\ell}^{*}: B^{*} \times A \longrightarrow B^{*} \text { and } \pi_{r}^{t * t}: A \times B^{*} \longrightarrow B^{*} .
$$

Thus $B^{*}$ is a left Banach $A$-module and a right Banach $A$-module with respect to the module actions $\pi_{r}^{t * t}$ and $\pi_{\ell}^{*}$, respectively. The the second dual $B^{* *}$ is a Banach $A^{* *}$-bimodule with the following module actions

$$
\pi_{\ell}^{* * *}: A^{* *} \times B^{* *} \longrightarrow B^{* *} \text { and } \pi_{r}^{* * *}: B^{* *} \times A^{* *} \longrightarrow B^{* *}
$$

where $A^{* *}$ is considered as a Banach algebra with respect to the first Arens product. Similarly, $B^{* *}$ is a Banach $A^{* *}$-bimodule with the module actions

$$
\pi_{\ell}^{t * * * t}: A^{* *} \times B^{* *} \longrightarrow B^{* *} \text { and } \pi_{r}^{t * * * t}: B^{* *} \times A^{* *} \longrightarrow B^{* *}
$$

where $A^{* *}$ is considered as a Banach algebra with respect to the second Arens product.

Let $B$ be a left Banach $A$-module and $e$ be a left unit element of $A$. Then $e$ is a left unit (resp. weakly left unit) for $B$, if $\pi_{\ell}(e, b)=b$ (resp. $\left\langle b^{\prime}, \pi_{\ell}(e, b)\right\rangle=\left\langle b^{\prime}, b\right\rangle$ for all $b^{\prime} \in B^{*}$ ) where $b \in B$. The definition of right unit (resp. weakly right unit) is similar. A Banach $A$-bimodule $B$ is called unital, if $B$ has the same left and right unit. In this way, $B$ is called a unitary Banach $A$-bimodule.

Suppose that $A$ is a Banach algebra and $B$ is a Banach $A$-bimodule. Since $B^{* *}$ is a Banach $A^{* *}$-bimodule, where $A^{* *}$ is equipped with the first Arens product, we define the topological center of the right module action of $A^{* *}$ on $B^{* *}$ as follows:

$$
\begin{aligned}
Z_{A^{* *}}^{\ell}\left(B^{* *}\right)=Z\left(\pi_{r}\right)= & \left\{b^{\prime \prime} \in B^{* *}: \text { the map } a^{\prime \prime} \rightarrow \pi_{r}^{* * *}\left(b^{\prime \prime}, a^{\prime \prime}\right): A^{* *} \rightarrow B^{* *}\right. \\
& \text { is } \left.\text { weak }^{*} \text {-weak }{ }^{*} \text { continuous }\right\}
\end{aligned}
$$

In this way, we write $Z_{B^{* *}}^{\ell}\left(A^{* *}\right)=Z\left(\pi_{\ell}\right), Z_{A^{* *}}^{r}\left(B^{* *}\right)=Z\left(\pi_{\ell}^{t}\right)$ and $Z_{B^{* *}}^{r}\left(A^{* *}\right)=$ $Z\left(\pi_{r}^{t}\right)$, where $\pi_{\ell}: A \times B \rightarrow B$ and $\pi_{r}: B \times A \rightarrow B$ are the left and right module actions of $A$ on $B$, for more information related to the Arens regularity of module actions on Banach algebras, see $[2,4,9,10]$. If we set $B=A$, we write $Z_{A^{* *}}^{\ell}\left(A^{* *}\right)=Z_{1}\left(A^{* *}\right)=Z_{1}^{\ell}\left(A^{* *}\right)$ and $Z_{A^{* *}}^{r}\left(A^{* *}\right)=Z_{2}\left(A^{* *}\right)=Z_{2}^{r}\left(A^{* *}\right)$, for more information see [12]. Let $A$ be a Banach algebra, $A^{*}$ and $A^{* *}$ be the first and second dual of $A$, respectively. For $a \in A$ and $a^{\prime} \in A^{*}$, by $a^{\prime} a$ and $a a^{\prime}$, we mean the functionals in $A^{*}$ defined by $\left\langle a^{\prime} a, b\right\rangle=\left\langle a^{\prime}, a b\right\rangle=a^{\prime}(a b)$ and $\left\langle a a^{\prime}, b\right\rangle=\left\langle a^{\prime}, b a\right\rangle=a^{\prime}(b a)$ for all $b \in A$, respectively. A Banach algebra $A$ is embedded in its second dual via the identification $\left\langle a, a^{\prime}\right\rangle-\left\langle a^{\prime}, a\right\rangle$ for every $a \in A$ and $a^{\prime} \in A^{*}$. 


\section{Main Results}

Consider the tensor product, $X \otimes Y$, of the vector space $X$ and $Y$ which can be constructed as a space of linear functional on $B(X \times Y)$. By $X \widehat{\otimes} Y$ we shall denote the projective tensor products of $X$ and $Y$, where $X \widehat{\otimes} Y$ is the completion of $X \otimes Y$ for the norm

$$
\|u\|=\inf \sum_{i=1}^{n}\left\|x_{i}\right\|\left\|y_{i}\right\|,
$$

where the infimum is taken over all the representations of $u$ as a finite sum of the form $u=\sum_{i=1}^{n} x_{i} \otimes y_{i}[5]$.

The natural multiplication of $A \widehat{\otimes} B$ is the linear extension of the following multiplication on decomposable tensors $(a \otimes b)(\tilde{a} \otimes \tilde{b})=a \tilde{a} \otimes b \tilde{b}$. For more details, see [16].

A functional $a^{\prime}$ in $A^{*}$ is said to be wap (weakly almost periodic) on $A$ if the mapping $a \rightarrow a^{\prime} a$ from $A$ into $A^{*}$ is weakly compact. Pym in [15] showed that this definition is equivalent with the following condition:

$$
\lim _{i} \lim _{j}\left\langle a^{\prime}, a_{i} b_{j}\right\rangle=\lim _{j} \lim _{i}\left\langle a^{\prime}, a_{i} b_{j}\right\rangle
$$

whenever both iterated limits exist, for any two net $\left(a_{i}\right)_{i}$ and $\left(b_{j}\right)_{j}$ in $\{a \in A$ : $\|a\| \leq 1\}$. The collection of all weakly almost periodic functionals on $A$ is denoted by $\operatorname{wap}(A)$. Also, $a^{\prime} \in \operatorname{wap}(A)$ if and only if $\left\langle a^{\prime \prime} b^{\prime \prime}, a^{\prime}\right\rangle=\left\langle a^{\prime \prime} o b^{\prime \prime}, a^{\prime}\right\rangle$ for every $a^{\prime \prime}, b^{\prime \prime} \in A^{* *}$. Thus, it is clear that $A$ is Arens regular if and only if $\operatorname{wap}(A)=A^{*}[9$, Theorem 2.6.17]. In the sequel, to show that the projective tensor products $A \widehat{\otimes} B$ is Arens regular, it is sufficient that we show that wap $(A \widehat{\otimes} B)=(A \widehat{\otimes} B)^{*}$. In all of this section, we regard $A^{*} \widehat{\otimes} B^{*}$ as a subset of $(A \widehat{\otimes} B)^{*}$ and by $A_{1}$ and $B_{1}$ we mean all elements of $a \in A$ and $b \in B$ such that $\|a\| \leq 1$ and $\|b\| \leq 1$.

Theorem 2.1. Suppose that $A$ and $B$ are Banach algebras and for every sequence $\left(x_{i}\right)_{i},\left(y_{j}\right)_{j} \subseteq A_{1},\left(z_{i}\right)_{i},\left(w_{j}\right)_{j} \subseteq B_{1}$ and $f \in B(A \times B)$, we have

$$
\lim _{j} \lim _{i} f\left(x_{i} z_{i}, y_{j} w_{j}\right)=\lim _{i} \lim _{j} f\left(x_{i} z_{i}, y_{j} w_{j}\right) .
$$

Then $A \widehat{\otimes} B$ is Arens regular.

Proof. Assume that $f \in B(A \times B)$. Since $B(A \times B)=(A \widehat{\otimes} B)^{*}$, it is enough to show that $f \in \operatorname{wap}(A \widehat{\otimes} B)$. Let $\left(x_{i}\right)_{i},\left(y_{j}\right)_{j} \subseteq A_{1}$ and $\left(z_{i}\right)_{i},\left(w_{j}\right)_{j} \subseteq B_{1}$, then we have the following equality

$$
\begin{aligned}
\lim _{j} \lim _{i}\left\langle f,\left(x_{i} \otimes y_{j}\right)\left(z_{i} \otimes w_{j}\right)\right\rangle & =\lim _{j} \lim _{i}\left\langle f, x_{i} z_{i} \otimes y_{j} w_{j}\right\rangle \\
& =\lim _{j} \lim _{i} f\left(x_{i} z_{i}, y_{j} w_{j}\right) \\
& =\lim _{i} \lim _{j} f\left(x_{i} z_{i}, y_{j} w_{j}\right) \\
& =\lim _{i} \lim _{j}\left\langle f,\left(x_{i} \otimes y_{j}\right)\left(z_{i} \otimes w_{j}\right)\right\rangle,
\end{aligned}
$$


for every $f \in(A \widehat{\otimes} B)^{*}$. This means that $f \in \operatorname{wap}(A \widehat{\otimes} B)$, and proof is complete.

Definition 2.1. Let $A$ be a Banach algebra and let $B$ be a Banach $A$-bimodule and let $\pi: A \widehat{\otimes} B \longrightarrow B$ such that $\pi(a \otimes b)=a b$ for every $a \in A, b \in B$. We say that $B$ is non-trivial on $A$, if $\pi$ is surjective and has a bounded right inverse.

Remark 2.1. In the above definition, if $A$ is unital, then $\pi$ will be surjective. Now, suppose $\pi$ has a continuous right inverse $\rho, e_{A}$ and $e_{B}$ are units of $A$ and $B$, respectively. Let $\varphi \in(A \widehat{\otimes} B)^{*}$, then $\varphi \circ \rho$ belongs to $B^{*}$. Hence, there is a $\phi \in B^{*}$ such that $\varphi \circ \rho=\phi$. In other word, in the following diagram, we have $\varphi \circ \rho=\phi \circ \operatorname{id}_{B}$.

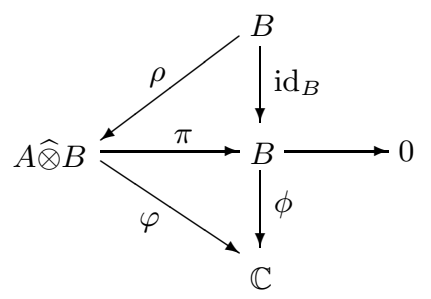

As well as, $\phi \circ \pi$ is in $(A \widehat{\otimes} B)^{*}$. Thus, there is a $\psi \in(A \widehat{\otimes} B)^{*}$ such that $\phi \circ \pi=\psi$. Then $\phi=\psi \circ \rho$. For given $a \otimes b \in A \widehat{\otimes} B$ we have

$$
\begin{aligned}
\phi \circ \pi(a \otimes b) & =\phi(a b)=\varphi \circ \rho(a b)=\varphi \circ \rho\left(e_{A} a e_{B} b\right) \\
& =\varphi \circ \rho\left(\left(e_{A} a\right)\left(e_{B} b\right)\right)=\psi \circ \rho\left(\left(e_{A} a\right)\left(e_{B} b\right)\right) \\
& =\psi(a \otimes b) .
\end{aligned}
$$

Then, by (2.1), for every $\varphi \in(A \widehat{\otimes} B)^{*}$ there is a $\psi \in(A \widehat{\otimes} B)^{*}$ such that $\varphi \circ \rho=\psi \circ \rho$ and $\psi(a \otimes b)=\psi \circ \rho(a b)$, for every $a \in A$ and $b \in B$. Since $A$ is unital, every element $c$ of $B$ can be written as $c=a b$ where $a \in A$ and $b \in B$. We can define $\rho: B \longrightarrow A \widehat{\otimes} B$ by $\rho(b)=e_{A} \otimes b$ and $\rho(a b)=\rho\left(\left(e_{A} a\right) b\right)=a \otimes b$, for every $a \in A$ and $b \in B$. By this definition $\rho$ is injective and it is a unique way to define of $\rho$. By this definition the above diagram commutes and we have $\phi \circ \pi(a \otimes b)=\varphi(a \otimes b)$, for every $a \in A$ and $b \in B$.

A wide class of Banach algebras which satisfy in the Definition 2.1, are projective and biprojective Banach algebras. A Banach algebra $A$-bimodule $B$ is called projective if $\pi: A^{\sharp} \widehat{\otimes} B \longrightarrow B$ has bounded right inverse in ${ }_{A} B\left(B, A^{\sharp} \widehat{\otimes} B\right)$ and the Banach algebra $A$ is called biprojective if $\pi: A \widehat{\otimes} A \longrightarrow A$ has bounded right inverse in ${ }_{A} B(A, A \widehat{\otimes} A$ ) (for more details see [18]).

Theorem 2.2. Let $A$ and $B$ be Banach algebras and $B$ is unital. Suppose $B$ is a Banach A-bimodule. Then

1. if $A \widehat{\otimes} B$ is Arens regular, then $A$ is Arens regular.

2. if $B$ is non-trivial on $A$ and $B$ be a unitary Banach $A$-bimodule. Then $A$ and $B$ are Arens regular if and only if $A \widehat{\otimes} B$ is Arens regular. 
Proof. 1. Assume that $A \widehat{\otimes} B$ is Arens regular and $u \in B$ is the unit element of $B$. We show that $\operatorname{wap}(A)=A^{*}$. Get $\left(a_{i}\right)_{i} \subseteq A,\left(c_{j}\right)_{j} \subseteq A$ and $a^{\prime} \in A^{*}$. Define $\phi=a^{\prime} \otimes b^{\prime}$ where $b^{\prime} \in B^{*}$ and $b^{\prime}(u)=1$. Since $A^{*} \otimes B^{*} \subseteq(A \widehat{\otimes} B)^{*}$ and $A \widehat{\otimes} B$ is Arens regular, we have $a^{\prime} \otimes b^{\prime} \in \operatorname{wap}(A \widehat{\otimes} B)$. Hence it follows that

$$
\begin{aligned}
\lim _{i} \lim _{j}\left\langle a^{\prime}, a_{i} c_{j}\right\rangle & =\lim _{i} \lim _{j}\left\langle a^{\prime} \otimes b^{\prime}, a_{i} c_{j} \otimes u\right\rangle \\
& =\lim _{i} \lim _{j}\left\langle a^{\prime} \otimes b^{\prime},\left(a_{i} \otimes u\right)\left(c_{j} \otimes u\right)\right\rangle \\
& =\lim _{j} \lim _{i}\left\langle a^{\prime} \otimes b^{\prime},\left(a_{i} \otimes u\right)\left(c_{j} \otimes u\right)\right\rangle \\
& =\lim _{j} \lim _{i}\left\langle a^{\prime}, a_{i} c_{j}\right\rangle .
\end{aligned}
$$

This means that $a^{\prime} \in \operatorname{wap}(A)$, and so $A$ is Arens regular.

2. Let $u$ be a unit element of $B$ and let $B$ be Arens regular. Then $\operatorname{wap}(B)=B^{*}$. Suppose that $\left(a_{i}\right)_{i} \subseteq A_{1}$ and $\left(b_{j}\right)_{j} \subseteq B_{1}$ whenever both iterated limits exist. Then $\left(a_{i} u\right)_{i} \subseteq B_{1}$, and so for every $b^{\prime} \in B^{*}$, we have the following equality

$$
\lim _{i} \lim _{j}\left\langle b^{\prime},\left(a_{i} u\right) b_{j}\right\rangle=\lim _{j} \lim _{i}\left\langle b^{\prime},\left(a_{i} u\right) b_{j}\right\rangle .
$$

Now; let $\varphi \in(A \widehat{\otimes} B)^{*}$. Then by Remark $2.1, \pi: A \widehat{\otimes} B \longrightarrow B$ has a continuous right inverse $\rho$ such that $\varphi \circ \rho$ belongs to $B^{*}$ and there is a $\phi \in B^{*}$ such that $\varphi \circ \rho=\phi$, and $\phi \circ \pi(a \otimes b)=\varphi(a \otimes b)$, for every $a \otimes b \in A \widehat{\otimes} B$. Now we have

$$
\begin{aligned}
\lim _{i} \lim _{j}\left\langle\varphi, a_{i} \otimes b_{j}\right\rangle & =\lim _{i} \lim _{j}\left\langle\phi \circ \pi, a_{i} \otimes b_{j}\right\rangle=\lim _{i} \lim _{j}\left\langle\phi, \pi\left(a_{i} \otimes b_{j}\right)\right\rangle \\
& =\lim _{i} \lim _{j}\left\langle\phi, a_{i} b_{j}\right\rangle=\lim _{i} \lim _{j}\left\langle\phi, a_{i}\left(u b_{j}\right)\right\rangle \\
& =\lim _{j} \lim _{i}\left\langle\phi,\left(a_{i} u\right) b_{j}\right\rangle=\lim _{j} \lim _{i}\left\langle\phi, \pi\left(a_{i} \otimes b_{j}\right)\right\rangle \\
& =\lim _{j} \lim _{i}\left\langle\varphi, a_{i} \otimes b_{j}\right\rangle .
\end{aligned}
$$

It follows that $\varphi \in \operatorname{wap}(A \widehat{\otimes} B)$, and so $A \widehat{\otimes} B$ is Arens regular. The converse by using the part (1) holds.

Corollary 2.1. Suppose that $A$ and $B$ are unital Banach algebras and $B$ is a unitary Banach A-bimodule. Assume that $B$ is non-trivial on $A$. If $A$ and $B$ are Arens regular, then every bilinear form $m: A \times B \rightarrow \mathbb{C}$ is weakly compact.

Proof. Apply Theorem 2.2 and Theorem 3.4 of [19].

Let $A$ and $B$ be Banach algebras. A bilinear form $m: A \times B \rightarrow \mathbb{C}$ is said to be biregular, if for any two pairs of sequence $\left(a_{i}\right)_{i},\left(\tilde{a}_{j}\right)_{j}$ in $A_{1}$ and $\left(b_{i}\right)_{i},\left(\tilde{b}_{j}\right)_{j}$ in $B_{1}$, we have

$$
\lim _{i} \lim _{j} m\left(a_{i} \tilde{a}_{j}, b_{i} \tilde{b}_{j}\right)=\lim _{j} \lim _{i} m\left(a_{i} \tilde{a}_{j}, b_{i} \tilde{b}_{j}\right)
$$


provided that these limits exist. There are some examples of biregular non regular bilinear form for more information see [19].

Corollary 2.2. Suppose that $A$ and $B$ are Banach algebras. Then we have the following assertions:

1. By the conditions of Theorem 2.1, every bilinear form $m: A \times B \rightarrow \mathbb{C}$ is biregular.

2. By the conditions of Theorem 2.2 (2), every bilinear form $m: A \times B \rightarrow \mathbb{C}$ is biregular.

In the following, we give a simple proof of Theorem 3.4 of [19].

Theorem 2.3. [19, Theorem 3.4] Let $A$ and $B$ be Banach algebras and $u: A \rightarrow B^{*}$ be a continuous linear operator. Then the bilinear form $m: A \times B \rightarrow \mathbb{C}$ defined by $m(a, b)=\langle u(a), b\rangle$ is biregular.

Proof. Let $\left(a_{i}\right)_{i},\left(\tilde{a}_{j}\right)_{j}$ in $A_{1}$ and $\left(b_{i}\right)_{i},\left(\tilde{b}_{j}\right)_{j}$ in $B_{1}$ such that the following iterated limits exist:

$$
\lim _{i} \lim _{j} m\left(a_{i} \tilde{a}_{j}, b_{i} \tilde{b}_{j}\right) \text { and } \lim _{j} \lim _{i} m\left(a_{i} \tilde{a}_{j}, b_{i} \tilde{b}_{j}\right) .
$$

There are $\left(a_{\alpha}\right)_{\alpha},\left(\tilde{a}_{\beta}\right)_{\beta}$ in $A$ and $\left(b_{\alpha}\right)_{\alpha},\left(\tilde{b}_{\beta}\right)_{\beta}$ in $B$ such that $a_{\alpha} \stackrel{w^{*}}{\rightarrow} a^{\prime \prime}$ and $\tilde{a}_{\beta} \stackrel{w^{*}}{\rightarrow} \tilde{a}^{\prime \prime}$ in $A^{* *}$ and we have $b_{\alpha} \stackrel{w^{*}}{\rightarrow} b^{\prime \prime}$ and $\tilde{b}_{\beta} \stackrel{w^{*}}{\rightarrow} \tilde{b}^{\prime \prime}$ in $B^{* *}$. Since $A$ and $B$ are Arens regular, we have

$$
\lim _{\alpha} \lim _{\beta} a_{\alpha} \tilde{a}_{\beta}=\lim _{\beta} \lim _{\alpha} a_{\alpha} \tilde{a}_{\beta}=a^{\prime \prime} \tilde{a}^{\prime \prime},
$$

and

$$
\lim _{\alpha} \lim _{\beta} b_{\alpha} \tilde{b}_{\beta}=\lim _{\beta} \lim _{\alpha} b_{\alpha} \tilde{b}_{\beta}=b^{\prime \prime} \tilde{b}^{\prime \prime} .
$$

Then, since $u$ is continuous, we have

$$
\begin{aligned}
\lim _{\alpha} \lim _{\beta} m\left(a_{\alpha} \tilde{a}_{\beta}, b_{\alpha} \tilde{b}_{\beta}\right) & =\lim _{\alpha} \lim _{\beta}\left\langle u\left(a_{\alpha} \tilde{a}_{\beta}\right), b_{\alpha} \tilde{b}_{\beta}\right\rangle \\
& =\left\langle u^{\prime \prime}\left(a^{\prime \prime} \tilde{a}^{\prime \prime}\right), b^{\prime \prime} \tilde{b}^{\prime \prime}\right\rangle .
\end{aligned}
$$

Similarly, we have

$$
\lim _{\beta} \lim _{\alpha} m\left(a_{\alpha} \tilde{a}_{\beta}, b_{\alpha} \tilde{b}_{\beta}\right)=\left\langle u^{\prime \prime}\left(a^{\prime \prime} \tilde{a}^{\prime \prime}\right), b^{\prime \prime} \tilde{b}^{\prime \prime}\right\rangle .
$$

Consequently, we have

$$
\lim _{i} \lim _{j} m\left(a_{i} \tilde{a}_{j}, b_{i} \tilde{b}_{j}\right)=\lim _{j} \lim _{i} m\left(a_{i} \tilde{a}_{j}, b_{i} \tilde{b}_{j}\right) .
$$

It follows that $m$ is biregular. 
Example 2.1. [19] Let $A$ be a Banach algebra and $1<p<\infty$. Then

1. $\ell^{p} \widehat{\otimes} A$ is Arens regular if and only if $A$ is Arens regular.

2. Let $G$ be a locally compact group. Then, $L^{p}(G) \widehat{\otimes} A$ is Arens regular if and only if $A$ is Arens regular.

Proof. For prove, we apply Theorem 3.4 of [19] and Theorem 2.7.

We finish this section with the following problems:

Problem 2.1. Let $G$ be a locally compact group. What can say for the following sets?

$$
Z_{L^{1}(G)^{* *}}^{\ell}\left(\left(L^{1}(G) \widehat{\otimes} L^{1}(G)\right)^{* *}\right)=? \quad Z_{L^{1}(G)^{* *}}^{\ell}\left(L^{1}(G)^{* *} \widehat{\otimes} L^{1}(G)^{* *}\right)=?
$$

\section{REF EREN C ES}

1. R. E. Arens, The adjoint of a bilinear operation, Proc. Amer. Math. Soc., 2(1951), 839-848.

2. A. Bagheri Vakilabad, K. Haghnejad Azar and A. Jabbari, Arens regularity of module actions and weak amenability of Banach algebras, Periodica Math. Hung., 71(2)(2015), 224-235.

3. J. Baker, A. T. Lau and J. S. Pym, Module homomorphism and topological centers associated with weakly sequentially compact Banach algebras, J. Func. Anal., 158(1998), 186-208.

4. A. Bodaghi, K. Haghnejad Azar and A. Jabbari, Some notes on the topological centers of module actions, Iran. J. Sci. Tech. (Sciences), 39(3.1)(2015), 417-431.

5. F. F. Bonsall and J. Duncan, Complete normed algebras, Springer-Verlag, Berlin 1973.

6. P. Civin and B. Yood, The second conjugate space of a Banach algebra as an algebra, Pacific J. Math., 11(1961), 820-847.

7. H. G. Dales, Banach algebra and automatic continuity, Oxford 2000.

8. H. G. Dales and A. T-M. Lau, The second dual of Beurling algebras, Mem. Amer. Math. Soc., 177 (2005).

9. M. Eshaghi Gordji and M. Filali, Arens regularity of module actions, Studia Math. 181(3)(2007), 237-254.

10. K. Haghnejad Azar, Arens regularity of bilinear forms and unital Banach module space, Bull. Iran. Math. Soc., 40(2)(2014), 505-520.

11. B. E. Johnson, Weak amenability of group algebras, Bull. London Math. Soc., 23(1991), 281-284.

12. A. T. Lau and A. Ülger, Topological center of certain dual algebras, Trans. Amer. Math. Soc., 348(1996), 1191-1212.

13. T. Miao, Unital Banach algebras and their subalgebras, Math. Proc. Camb. Phil. Soc., 143(2007), 343-347. 
14. S. Mohamadzadeh and H. R. E. Vishki, Arens regularity of module actions and the second adjoint of a derivation, Bull. Aust. Math. Soc., 77(2008), 465-476.

15. J. S. Pym, The convolution of functionals on spaces of bounded functions, Proc. London Math. Soc., 15(1965), 84-104.

16. R. A. Rayan, Introduction to tensor product of Banach plgebras, Springer-Verlag, London 2002.

17. Reiter, H., Stegeman, J. D., Classical harmonic analysis and locally compact groups, London Math. Soc. Mono. Ser. Volume 22, Oxford, 2000.

18. V. Runde, Lectures on Amenability, Springer, New York 2002.

19. A. Ülger, Arens regularity of the algebra $A \widehat{\otimes} B$, Trans. Amer. Math. Soc., 305(2)(1988), 623-639.

20. A. Ülger, Arens regularity of weakly sequentialy compact Banach algebras, Proc. Amer. Math. Soc., 127(11)(1999), 3839-3839.

21. A. Ülger, Erratum to Arens regularity of the algebra $A \widehat{\otimes} B$, Trans. Amer. Math. Soc., 355(9)(2003), 623-639.

Mostfa Shams Kojanaghi

Department of Mathematics

Islamic Azad University Tehran

Tehran, Iran

mstafa.shams99@yahoo.com

Kazem Haghnejad Azar

Faculty of Science

Department of Mathematics

University of Mohaghegh Ardabili, Ardabil, Iran

Ardabil, Iran

haghnejad@uma.ac.ir 